\title{
COVID-19-Related Acute Ischemic Stroke in Young Adults: What Is the Optimal Antithrombotic Regimen for Secondary Prevention?
}

\author{
Fahimeh Vahabizad ${ }^{\mathrm{a}}$, Maryam Sharifian Dorche ${ }^{\mathrm{b}, \mathrm{c}}$, Pegah Mohammadi ${ }^{\mathrm{d}}$, \\ Kasra Khatibie , Ashkan Mowla, $\mathrm{g}$
}

\section{To the Editor}

Acute ischemic stroke (AIS) has been reported as a serious neurological complication after severe acute respiratory syndrome coronavirus 2 (SARS-CoV-2) infection. It was shown that occurrence of AIS in coronavirus disease -19 (COVID-19) was correlated with the severity of respiratory illness [1,2].

Despite many reports of AIS and COVID-19 in older patients with established cardiovascular risk factors, there are several reports of AIS in young patients without any significant past medical history or cardiovascular risk factors $[2,3]$. Such reports suggest hypercoagulability and/or endothelial dysfunction within the arteries of the COVID-19 patients. Furthermore, it has been shown that inflammation and hypercoagulable processes contribute to developing both venous and arterial thromboembolism following infection with SARS-CoV-2 [1, 4-7]. These potential mechanisms as well as the clinical and epidemiological differences in patients with COVID-19 compared to non-COVID-19 patients, raise the question of the optimal secondary stroke prevention antithrombotic regimen in young COVID-19-related AIS patients.

To address such an important question, there is need for randomized clinical trials and high-quality prospective studies

Manuscript submitted July 16, 2020, accepted July 23, 2020

Published online August 2, 2020

${ }^{\text {aDepartment }}$ of Neurology, Sina Hospital, Tehran University of Medical Sciences, Tehran, Iran

${ }^{\mathrm{b}}$ Clinical Neurology Research Center, Shiraz University of Medical Sciences, Shiraz, Iran

'Department of Neurology and Neurosurgery, Montreal Neurological Institute, McGill University, Montreal, Quebec, Canada

${ }^{\mathrm{d} C a l i f o r n i a}$ Institute of Neuroscience, Thousand Oaks, CA 91360, USA

eDepartment of Radiology, David Geffen School of Medicine, University of California Los Angles, Los Angeles, CA, USA

fDepartment of Neurological Surgery, Keck School of Medicine, University of Southern California (USC), Los Angeles, CA, USA

${ }^{g}$ Corresponding Author: Ashkan Mowla, Division of Endovascular Neurosurgery, Department of Neurological Surgery, Keck School of Medicine, University of Southern California (USC), 1200 North State St. Suite 3300, Los Angeles, CA 90033, USA. Email: mowla@usc.edu

doi: https://doi.org/10.14740/jnr616 with reasonable duration of follow-up. However, given lack of such studies to date, we aimed to review the limited current literature to investigate the optimal antithrombotic regimen for secondary prevention strategy in this group of patients.

We carried out a review in PubMed to find all articles evaluating secondary prevention of AIS following COVID-19 from December 1, 2019 to June 30, 2020. The keywords: "COVID-19" or "SARS-CoV-2 "or "coronavirus" and "stroke" or" cerebrovascular" and "treatment "or "secondary prevention" were used in different combinations. All pertinent case reports, case series and original research articles in English language were included.

The literature search revealed 430 articles. After eliminating the duplications and non-relevant articles, seven articles were included in the study. We identified only the patients with COVID-19-related AIS who did not have any pertinent past medical history or cardiovascular risk factor and were 50 years old or younger.

Data for secondary prevention antithrombotic regimen in 16 patients with above-mentioned inclusion criteria were available. Mean age of the patients was 39.5 years (range: 31 - 50). Anticoagulation was administered for $10(62.5 \%)$, single antiplatelet for three (18.7\%) and dual antiplatelet for three (18.7\%) patients (Table 1) [2-4, 8-11].

Despite the fact that several studies have shown the possibility of hypercoagulable state in the young COVID-19 patients, so far, no consensus on the secondary prevention antithrombotic regimen exists. Furthermore, the optimal secondary prevention measures in this particular group of patients might differ from the usual secondary prevention strategies in AIS patients with established cardiovascular risk factors [12].

Based on the findings of this review, it appears that there is a tendency toward utilizing anticoagulation rather than antiplatelets for secondary stroke prevention in this cohort of patients [2-4]. However, the efficacy of such approach for secondary prevention remains unclear given absence of long term follow ups and limited duration of the COVID-19. Furthermore, risk of intracranial hemorrhage (ICH) following administration of anticoagulation in the acute phase of AIS should be weighed against its potential benefit in these patients.

To better identify the optimal antithrombotic regimen for secondary prevention in COVID-19-related AIS, it is impor- 


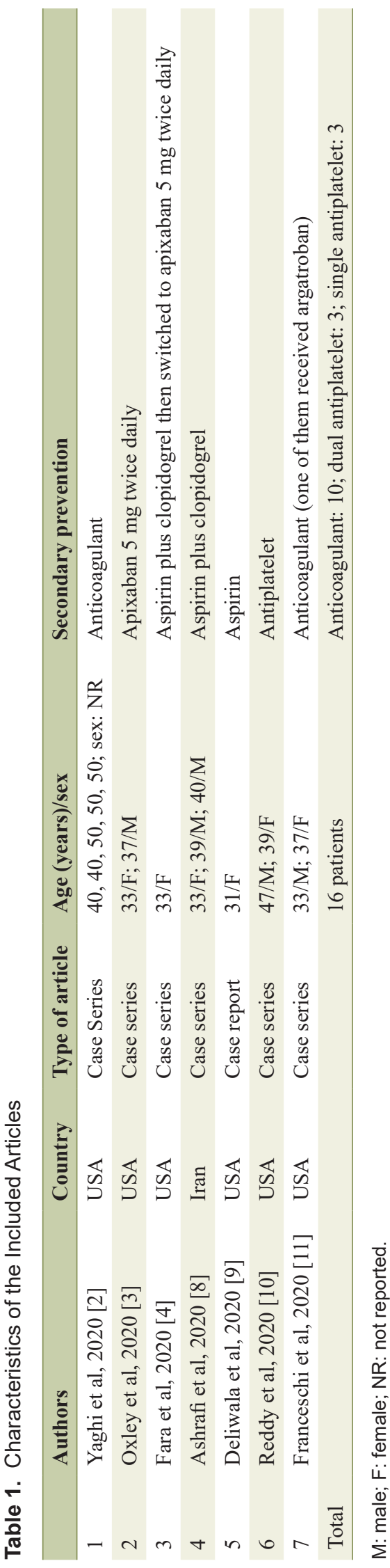

tant to better understand the underlying mechanisms of such strokes. The potential mechanisms playing a role in such pathology are reviewed below.

\section{Hyper-coagulable state}

There is a high incidence of thrombotic events reported in critically ill patients with COVID-19 which is thought to be out of proportion to the underlying systemic inflammatory state. A major underlying etiology of such hypercoagulable state has been shown to be secondary consumptive coagulopathy evident by marked elevation in D-dimer, lactate dehydrogenase (LDH) and drop in fibrinogen levels in severe COVID-19 patients [13]. This may contribute to COVID19-related AIS.

\section{Inflammation}

SARS-CoV-2 has been shown to trigger a severe inflammatory response. Such an inflammatory state could lead to instability of the atherosclerotic plaque susceptible to rupture. Marked elevation of C-reactive protein (CRP), interleukin 6 (IL-6) and IL-7 are supportive evidence of pro-inflammation process in COVID-19 patients $[14,15]$. This mechanism may not necessarily apply to the patients we enrolled in this study as they might not have any underling atherosclerotic plaque.

\section{Cardiac involvement}

Cardiac involvement can be the other potential mechanism that might link the COVID-19 with AIS. Myocarditis, arrhythmias and hypotension as complications of COVID-19 can contribute to cerebral hypo-perfusion and subsequently AIS [16].

\section{Angiotensin-converting enzyme (ACE)}

Several studies have documented that ACE2 has a significant role in renin angiotensin system (RAS) and consequently the balance of ACE1 and angiotensin II levels. SARS-CoV-2 virus binds to ACE2 which is in the lung, small intestinal and brain endothelial cells. Depletion of ACE2 by SARS-CoV-2 virus might cause an imbalance in the RAS which might result in endothelial dysfunction and subsequently AIS [17].

\section{Direct invasion of virus}

The virus could potentially cause a vasculitis process by infiltration of inflammatory cells and local necrosis and even direct spread to the vessel wall [18].

Patients with COVID-19 who suffer AIS and have a known underlying mechanism for AIS (e.g., atrial fibrillation) would still warrant the evidence-based antithrombotic regimen based on the current American Heart Association/American Stroke 
Association (AHA/ASA) guidelines for secondary stroke prevention, but the challenge on what antithrombotic is optimal for secondary stroke prevention remains with those patients who do not have any cerebrovascular risk factors or well-known underlying mechanisms for AIS and their strokes thought to be associated with the COVID-19 procoagulant state. Furthermore, given COVID-19 has a monophasic course, it is still unclear how long the antithrombotic agent should continue for secondary prevention after AIS.

\section{Conclusions}

Based on the findings of this study, it seems that there is a tendency toward utilizing anticoagulants rather than antiplatelets for secondary stroke prevention in COVID-19-related AIS among health care providers. Till more robust data is available on this matter with longer follow ups, the decision regarding what antithrombotic agent is optimal should be individualized with taking into consideration the overall clinical and radiological features of the patient and also weighing the potential risks versus intended benefits of the antithrombotic agents. Ideal duration of the secondary prevention antithrombotic regimen also needs further investigation.

\section{Acknowledgments}

None to declare.

\section{Financial Disclosure}

None to declare.

\section{Conflict of Interest}

The authors declare that they have no relevant conflict of interest to report.

\section{Informed Consent}

Not applicable.

\section{Author Contributions}

All authors have contributed to the theoretical formalism, designing the study, data collection, data analysis and writing the manuscript.

\section{Data Availability}

The authors declare that data supporting the findings of this study are available within the article.

\section{References}

1. Mao L, Jin H, Wang M, Hu Y, Chen S, He Q, Chang J, et al. Neurologic manifestations of hospitalized patients with coronavirus disease 2019 in Wuhan, China. JAMA Neurol. 2020.

2. Yaghi S, Ishida K, Torres J, Mac Grory B, Raz E, Humbert $\mathrm{K}$, Henninger $\mathrm{N}$, et al. SARS-CoV-2 and stroke in a New York healthcare system. Stroke. 2020;51(7):20022011.

3. Oxley TJ, Mocco J, Majidi S, Kellner CP, Shoirah H, Singh IP, De Leacy RA, et al. Large-vessel stroke as a presenting feature of COVID-19 in the young. N Engl J Med. 2020;382(20):e60.

4. Fara MG, Stein LK, Skliut M, Morgello S, Fifi JT, Dhamoon MS. Macrothrombosis and stroke in patients with mild Covid-19 infection. J Thromb Haemost. 2020.

5. Shakibajahromi B, Borhani-Haghighi A, Haseli S, Mowla A. Cerebral venous sinus thrombosis might be underdiagnosed in the COVID-19 era. Version 2. eNeurologicalSci. 2020;20:100256.

6. Mowla A, Doyle J, Lail NS, Rajabzadeh-Oghaz H, Deline C, Shirani P, Ching M, et al. Delays in door-to-needle time for acute ischemic stroke in the emergency department: A comprehensive stroke center experience. J Neurol Sci. 2017;376:102-105.

7. Nabavizadeh SA, Mowla A, Mamourian AC. Wallerian degeneration of the bilateral middle cerebellar peduncles. J Neurol Sci. 2015;349(1-2):256-7.

8. Ashrafi F, Zali A, Ommi D, Salari M, Fatemi A, ArabAhmadi M, Behnam B, et al. COVID-19-related strokes in adults below 55 years of age: a case series. Neurol Sci. 2020;41(8):1985-1989.

9. Deliwala S, Abdulhamid S, Abusalih MF, Al-Qasmi MM, Bachuwa G. Encephalopathy as the sentinel sign of a cortical stroke in a patient infected with coronavirus disease-19 (COVID-19). Cureus. 2020;12(5):e8121.

10. Reddy ST, Garg T, Shah C, Nascimento FA, Imran R, Kan P, Bowry R, et al. Cerebrovascular disease in patients with COVID-19: a review of the literature and case series. Case Rep Neurol. 2020;12(2):199-209.

11. Franceschi AM, Arora R, Wilson R, Giliberto L, Libman RB, Castillo M. Neurovascular complications in COVID-19 infection: Case Series. AJNR Am J Neuroradiol. 2020.

12. Powers WJ, Rabinstein AA, Ackerson T, Adeoye OM, Bambakidis NC, Becker K, Biller J, et al. Guidelines for the early management of patients with acute ischemic stroke: 2019 update to the 2018 guidelines for the early management of acute ischemic stroke: a guideline for healthcare professionals from the American Heart Association/American Stroke Association. Stroke. 2019;50(12):e344-e418.

13. Lodigiani C, Iapichino G, Carenzo L, Cecconi M, Ferrazzi $\mathrm{P}$, Sebastian T, Kucher N, et al. Venous and arterial thromboembolic complications in COVID-19 patients admitted to an academic hospital in Milan, Italy. Thromb Res. 2020;191:9-14. 
14. Mohamud AY, Griffith B, Rehman M, Miller D, Chebl A, Patel SC, Howell B, et al. Intraluminal carotid artery thrombus in COVID-19: another danger of cytokine storm? AJNR Am J Neuroradiol. 2020.

15. Raucci F, Mansour AA, Casillo GM, Saviano A, Caso F, Scarpa R, Mascolo N, et al. Interleukin-17A (IL17A), a key molecule of innate and adaptive immunity, and its potential involvement in COVID-19-related thrombotic and vascular mechanisms. Autoimmun Rev. 2020;19(7):102572.
16. Kochi AN, Tagliari AP, Forleo GB, Fassini GM, Tondo C. Cardiac and arrhythmic complications in patients with COVID-19. J Cardiovasc Electrophysiol. 2020;31(5):1003-1008.

17. Goldberg MF, Goldberg MF, Cerejo R, Tayal AH. Cerebrovascular Disease in COVID-19. AJNR Am J Neuroradiol. 2020;41(7):1170-1172.

18. Morassi M, Bagatto D, Cobelli M, D'Agostini S, Gigli GL, Bna C, Vogrig A. Stroke in patients with SARS-CoV-2 infection: case series. J Neurol. 2020;267(8):2185-2192. 